\title{
Hacia la convergencia total de las tecnologías de la información
}

\author{
L.R. Vega-González \\ Centro de Ciencias A plicadas y Desarrollo Tecnológico, CCADET \\ Coordinación de Vinculación, Laboratorio de Cibernética y Sistemas \\ E-mail: Irvg@servidor.unam.mx
}

(recibido: octubre de 2003; aceptado: abril de 2004)

\begin{abstract}
Resumen
Diversos estudios indican que la dinámica de la innovación tecnológica ha crecido en forma exponencial durante los últimos cincuenta años, impulsada aparentemente por los avances sustanciales y continuos de las tecnologías de la información (Tl's). La disponibilidad de información con bajo costo relativo, en cualquier momento y en cualquier lugar, ha propiciado que se identifique a la actual sociedad como "sociedad de la información", la cual está evolucionando hacia la "sociedad del conocimiento". Este es y será, tal vez uno de los fenómenos más importantes y de mayor impacto en las décadas por venir. En este trabajo se presentan los primeros resultados de un estudio sobre la evolución de las tecnologías de la información que dieron origen y propiciaron el fenómeno de la globalización económica. La prospectiva indica que en algunas décadas se dará la convergencia absoluta de las TI's. De esta manera, parece muy probable que el continuo avance de tres tecnologías fundamentales, los dispositivos semiconductores (S), los sistemas de cómputo (C) y de los protocolos de comunicaciones $(\mathrm{P})$, con sus diferentes impactos, transformará definitivamente la vida del ser humano, sus organizaciones y sus sociedades. El fenómeno plantea la problemática y la necesidad de generar un sistema de adaptación individual y colectiva que sea capaz de responder adecuadamente ante la dinámica de los cambios.
\end{abstract}

Descriptores: Tecnologías de la información, convergencia, globalización, impactos.

\begin{abstract}
Several worksin di catethat thetech nologi cal in nova tion dy nam icshasgrown exponent ially during thelast fifty years, ap par ently driven by thesub stan tially con tin u ous ad vances in the infor ma tion tech nol ogy (IT). The low cost in for ma tion avail abil ity in any place, and in any moment, has propi ti ated theiden ti fi ca tion of theactual so ci ety as the "in for ma tion soci ety", which in turn has been trans formed to the "knowl edgeso ci ety". This will may be the most im por tant and ma jor im pact ing phenomen on in thefol lowing decades. In this work, we pres ent preliminary results of an IT evo lu tion ary study, sup port ing thefact that they gaveor $i$ gin and actu ally support the eco nomic globalisation pro cess. In such a way, it seems very prob a bly that the con tin uousad vancemen tof threefun da men tal in for ma tion tech nol ogy ty pes, semi con ductor devi ces (S), Com puter Systems $(C)$, and $C$ om mu ni ca tion proto cols $(P)$, with their differ ent im pacts will de fin i tively transform hu man being life, itsor ga niza tions and soci eties. Finally, theprob lem of the need of an ad ap ta tion system for ad equatein di vid ual and col lectiveresponsein front of the dynamic changes is ad dressed.
\end{abstract}

Keywords: In for mation tech nol ogy con ver gence, globalisation, im pacts. 


\section{El entorno tecnológico en la primera década del Siglo XXI}

El cambio tecnológico ha sido el propulsor de la situación que hoy en día guarda el sistema socioeconómico y político mundial, así como de los diversos impactos que se perciben en las organizaciones y en los individuos. En sus trabajos sobre los ciclos económicos, Modelski (2001), señala que la onda No. 19 de Kondratieff, dio inicio con la aparición de Internet a finales de los setentas y que llegará hasta el año 2020 cubriendo el promedio de cincuenta años que toman los ciclos de innovación. A todas luces parece, que du rante los años que perdure este ciclo económico, el desarrollo tecnológico de las comunicaciones será impresionante, dando lugar a sistemas muy sofisticados que serán desarrollados con la aparente intención de mejorar el nivel de vida de los individuos, pero dando lugar también, a una gran acumulación de capitalque va a generar un mayor aumento en los niveles de pobreza.

Esta contradicción es muy clara para Dubois, quien citado en la revista Fiap (1998), considera que en el desarrollo de la economía global intervienen tanto las tecnologías como las ideologías, por lo que en un nuevo mundo global será necesario eliminar la marginación del hombre y respetar la naturaleza. El problema es, que las innovaciones que no respeten estos principios no darán paso a una revolución, sino a una involución.

La era de la información se reconoce como tal a partir del descubrimiento del transistor por los Laboratorios Bell en 1951, ya que du rante las décadas siguientes, las diversas tecnologías relacionadas con el uso de los dispositivos semiconductores para el manejo de las comunicaciones sufrieron una evolución extraordinaria y constante. En un principio, la ola de innovación se dio en el hardware de los sistemas, lográndose sistemas muy poderosos y eficientes a partir de la integración de los procesadores, las memorias y los periféricos. Aunado a esto, se desarrolló software operativo de alta eficiencia y confiabilidad, el cual estuvo disponible en diversas versiones a fina les del siglo XX.

Por otra parte, el incremento en el negocio de las empresas que ofrecían el servicio de transporte de señales telefónicas, "carriers", propició el desarrollo del software especializado en comunica- ciones. Las diferentes versiones de este software son conocidas como protocolos de comunicación.

Los protocolos permitieron el establecimiento de redes de computadoras usadas inicialmente con fines bélicos y posteriormente para facilitar y mejorar el manejo de datos, imágenes y audio a través de los multimedios, haciendo uso de redes de comunicación satelital con medios de transmisión de fibra óptica de banda ancha. Este nuevo esquema disminuyó los costos de las comunicaciones y mejoró la calidad del servicio.

De tal manera que podemos afirmar, que el desarrollo de distintas tecnologías dirigido hacia la mejora de las comunicaciones, generó el fenómeno conocido como convergencia de las tecnologías de la información. Como explicaremos más adelante, es la razón de que hoy en día sea posible establecer comunicaciones prácticamente instantáneas entre dos sitios opuestos en el mundo. Este alarde tecnológico dio sustento y factibilidad a las transacciones comerciales y financieras múltiples, derrumbando las barreras comerciales. La situación fue aprovechada, y tal vez provocada, por los impulsores de las corrientes económicas neoliberales para dar forma a un nuevo esquema geopolítico global sin fronteras físicas.

En los siguientes apartados haremos una breve reseña de la forma en que han evolucionado du rante las últimas décadas las tecnologías de la información que mayormente han contribuido para formar al entorno tecnológico global que existe en la actualidad. También se presentarán las tecnologías que se espera cobrarán una importancia es pe cial en el fu turo próximo. Todo parece indicar que la conformación de la situación futura del sistema mundial estará definida por el desarrollo que sufran los sistemas de comunicación, los cuales serán cada vez más abiertos y envolventes.

\section{La evolución de los dispositivos electrónicos}

\author{
El tran sistor y los circuitos de alta escala de \\ integración
}

La primera versión del bulbo o válvula electrónica de vacío, fue patentada en 1904 por Flemming. Dos años más tarde, fue mejorado y patentado por Lee de Forrest, en su versión de tríodo. Este 
dispositivo fue aplicado en una gran diversidad de aparatos, destacando los grandes sistemas de conmutación telefónica y los sistemas de radar tan exitosos en la segunda guerra mundial. El tran sistor a su vez, fue el resultado de un proyecto de investigación y desarrollo que buscaba un sustituto para las distintas versiones de bulbos.

La motivación de la investigación fue la de obtener dispositivos que requirieran menor consumo de potencia en su operación, ya que los sistemas de conmutación telefónica de la época consumían grandes cantidades de kilowatts, lo que hacia muy costosa su operación. En menos de diez años, los transistores de silicio y de germanio habían reemplazado los bulbos en casi todas sus aplicaciones.

En la década de los cincuenta, se presentó un problema muy serio en la manufactura de circuitos electrónicos que usaban transisto res para diversas aplicaciones: su alambrado e interconexión. Este problema se convirtió en un factor básico que afectaba el costo y la confiabilidad de los equipos. Así fue como se motivó el desarrollo del circuito integrado monolítico.

Sorprendentemente, llevar a la práctica la idea de integrar varios transistores en un solo semiconductor tomó trece años. El circuito integrado fue inventado independientemente y en paralelo por Jack Kilby de Texas In stru ments y por Rob ert Noyce de Fairchild en 1958 (Smith y Antoniadis, 1990).

Los fabricantes de semiconductores evaluaron rápidamente la potencialidad de la invención, por lo que en 1964, Gordon Moore, quien fue uno de los fundadores de Intel en 1968, predijo que la densidad de integración de circuitos se duplicaría cada año. Esta predicción resultó ser muy exacta, por lo que después sería considerada como la "ley de Moore".

A partir de entonces se desarrollaron fábricas de semiconductores basados en silicio. Para finales de los años sesenta, los circuitos de "gran escala de integración" (Large Scale Integration, LSI), estaban listos.

A finales de los setenta los fabricantes ya contaban con circuitos de "escala de integración muy grande", (Very Large Scale In te gration, VLSI)y a finales de los ochenta con dispositivos de la llamada ultra alta escala de integración (Ultra-Large Scale In te gra tion, ULSI). Los chips ULSI son hoy en día los módulos de construcción básica de todos los dispositivos electrónicos modernos, tales como radios, TV, sistemas de telefonía, radar, computadoras, y en general, productos electrónicos caseros e industriales.

El objetivo cen tral del desarrollo tecnológico en semiconductores, era el disminuir los requerimientos de potencia en las nuevas aplicaciones. Esto se cumplió a plenitud, además se ahorró espacio y disminuyeron los costos de fabricación. En la figura 1 se muestra la evolución de los procesadores en términos de la capacidad de transistores que tiene cada oblea semiconductora o chip.

Durante finales de los años ochenta y los noventa, el problema de espacio y consumo ya estaba resuelto, pero las empresas buscaron nuevas formas de optimización de los dispositivos electrónicos. La naturaleza del proceso del desarrollo de semiconductores cambió sustancialmente. Recientemente se han elaborado dispositivos que aprovechan el "efecto cuántico" basados en nuevas aleaciones de Arseniuro de Galio (AsGa), tales como el MODFET y el PRESTFET (Smith $\&$ Antoniadis, OP Cit).

\section{Evolución de la memoria de acceso aleatorio} (RAM)

Los computadores no hubieran podido evolucionar contando sólo con la tecnología de los nuevos procesadores, también fue necesaria la evolución de los chips de memoria, por lo que los fabricantes de semiconductores participaron activamente en este desarrollo.

El mercado de semiconductores fue dominado desde un principio por los fabricantes de los Estados Unidos de Norteamérica, y continuó así du rante las dos primeras décadas; sin em bargo, en los años ochenta se manifestó la presencia de nuevos fabricantes de semiconductores en Asia.

Un grupo de compañías japonesas encabezadas por Hitachi, NEC y Toshiba obtuvieron gran ventaja en el desarrollo de nuevas tecnologías de producción e invirtieron en tecnologías de integración y nuevas capacidades de manufactura. Según lansati (1997), un grupo de científicos e ingenieros Koreanos educados en los Estados Unidos de Norteamérica, regresaron a sus paises con el último conocimiento en litografía y diseño de transistores, por lo que construyeron la base tecnológica y científica que ayudó a compañías como la Samsung Koreana a ser líder en el mercado de memorias dinámicas (DRAM's) a principios de los años noventa. 


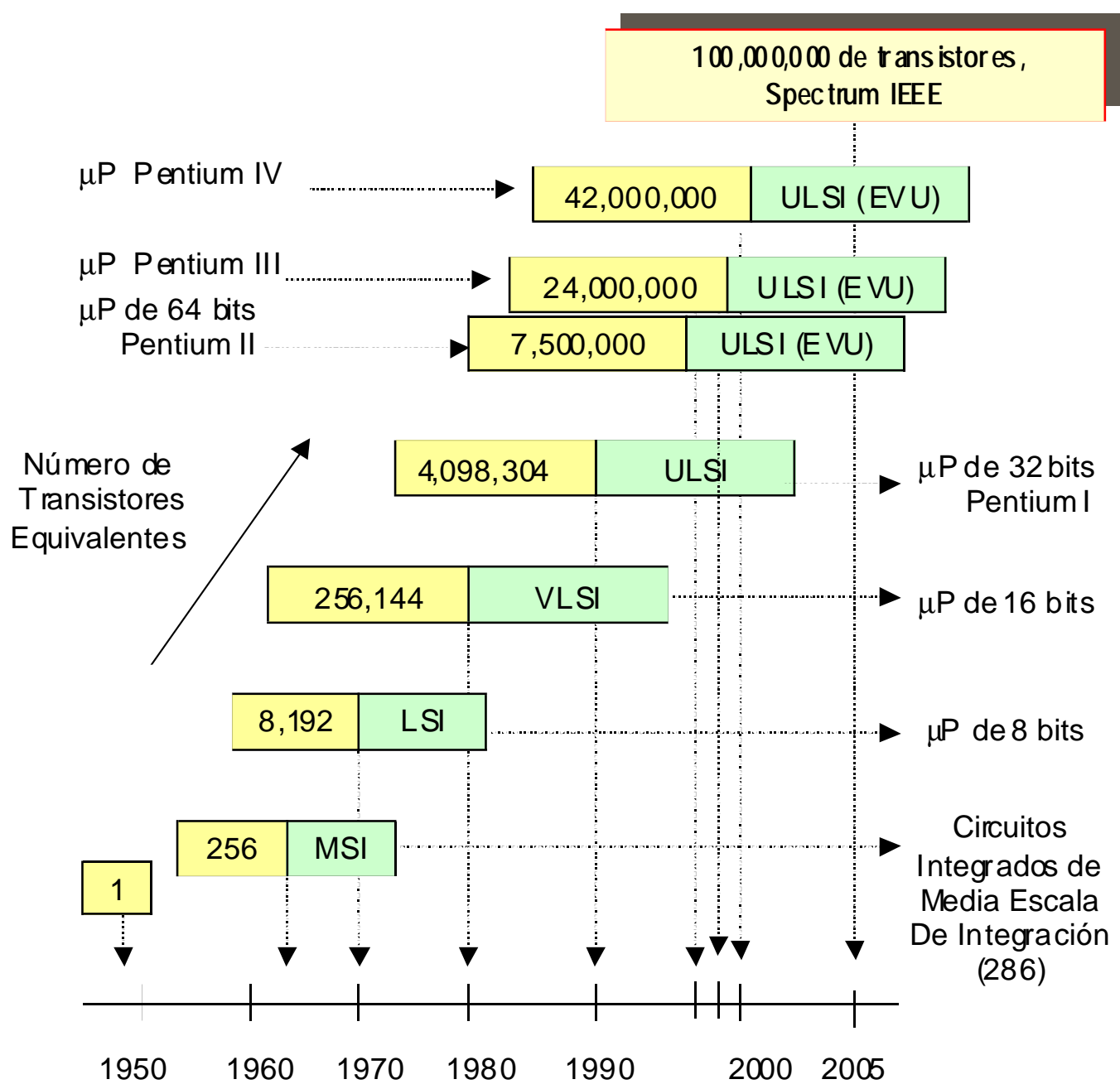

Figura 1. Evolución en el tiempo de las Escalas de Integración de los Semiconductores. (Elaboración propia)

En la figura 2 se muestra cómo la dinámica del mercado impulsó la evolución de la capacidad de las memorias RAM de menos de 1 MB por chip en 1988, a chips de RAM dinámica con capacidad de 32 y 64 MB por chip en menos de una década.

El costo de una instalación de tamaño mínimo para la fabricación de chips de memoria, se incrementó de 4 millones de dólares en 1971 a más de 1200 millones en 1996, y se incrementó a más de 4000 millones en el 2001.

\section{Evolución de la computadora}

\section{La computadora mainframe}

La computadora electrónica ENIAC fue desarrollada en 1950 por la Universidad de Pensilvania EUA. Ideada para realizar cálculos matemáticos sofisticados, en algunas décadas se convertiría en un dispositivo de cálculo de aplicación universal. Los fabricantes visualizaron un gran potencial en el mercado empresarial y pronto orientaron el hardware y soft ware de los computadores a la ejecución de programas para aplicaciones empresariales, administrativas, contables, de control de personal, con trol de la producción e inventarios que requerían el manejo de grandes volúmenes de información.

Una de las primeras aplicaciones de los chips VLSI en las computadoras electrónicas, fue el desarrollo de sistemas de procesamiento de datos de tarjetas múltiples o Unidades de Procesamiento Cen tral (CPU's). Esto dio origen a la aparición en el mercado de equipos grandes y poderosos, conocidos como computadores "mainframe" de gran escala. Estos equipos en sus primeras etapas se aplicaron a la solución de tareas administrativas sin descuidar su aplicación a la investigación en las universidades e institutos. 


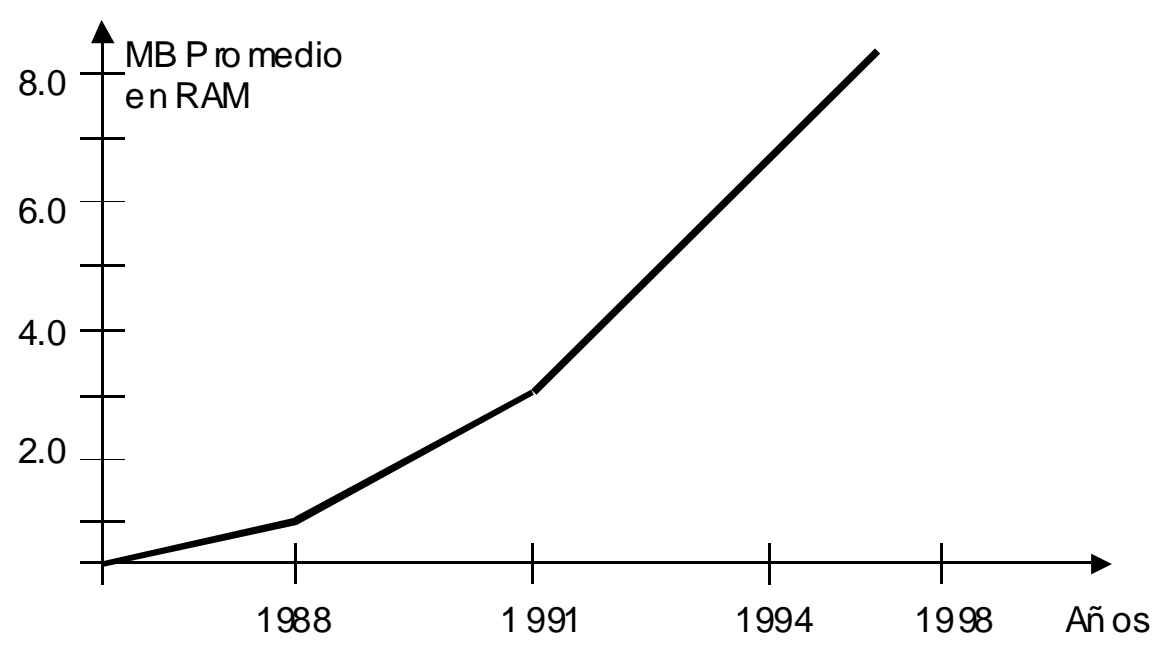

Figura 2. Crecimiento promedio del tamaño de las memorias RAM (O DISSEO-DIS, Facultad de Ingeniería de la Universidad de Nápoles, Federico II, (Esposito y Mastroiani, 1998)

La computadora mainframe logró un tremendo éxito de mercado, lo que hizo a IBM una de las corporaciones internacionales más ricas y poderosas entre 1960 y 1980.

En esa época, las primeras versiones de los programas de aplicación consistían de miles de líneas de instrucciones, desarrolladas en programas poco eficientes, entonces para que los fabricantes de computadoras se mantuvieran competitivos, fue esencial desarrollar computadoras más veloces en cuanto al procesamiento de las instrucciones de sus programas.

Así se inició la carrera de la velocidad de procesamiento, tratando de ser el líder en esta guerra mercantil. Texas Instruments patentó en 1970 el primer micro procesador de silicio. Este dispositivo era un procesador de datos monolítico embebido en una sola oblea semiconductora, el cual sería aplicado a los sistemas de cómputo de la empresa.

El manejo de información masiva planteaba otro problema: ¿dónde y cómo almacenar los datos que se procesaban?; es decir, el problema de la memoria temporal y fija. Las primeras memorias utilizadas en computadores híbridos fueron de ferrita magnética de baja velocidad y alto consumo de potencia. En 1969, Intel introdujo el primer chip de memoria con capacidad de almacenamiento de 1024 bits.

La tecnología de semiconductores ya estaba en el mercado; sin embargo, los fabricantes de equipos de cómputo integrados tardaron un promedio de entre 5 y 10 años para asimilar las tecnologías de microprocesadores y memorias.
Fue hasta mediados de los años setenta y principios de los ochenta que lograron la optimización de los primeros computadores electrónicos. Los microprocesadores usados en esas computadoras sustituyeron las tecnologías mecánicas de válvulas de vacío, de transistores e híbrida, usadas previamente.

Hacia finales de los setenta, al difundirse la aplicación de circuitos integrados de alta escala de integración, aparecieron los primeros computadores electrónicos dedicados, los cuales estaban destinados a realizar una aplicación específica. Los sistemas de telefonía de esa época son un buen ejemplo, ya que prácticamente eran computadoras digitales especializadas. Lo mismo sucedió con los sistemas de instrumentación y control, los sistemas para transmisión de radio y TV de la época, los sistemas aplicados a la medicina, la manufactura y el entretenimiento, entre muchos otros.

A principios de los ochenta, las líneas telefónicas enlazaban computadoras. Las industrias del computo y telefonía empezaban a entrelazarse.

\section{La computadora personal}

IBM dejo de ser el líder tecnológico en los noventas, sus directivos fallaron en sus pronósticos. Ellos consideraron que el futuro de la computación a escala mundial estaba en los grandes sistemas de cómputo mainframe de usuarios múltiples y no en las computadoras individuales.

Sus pronósticos se sustentaban en la percepción del mercado que dominaban y no en un 
monitoreo que ayudara a predecir el futuro de las tecnologías fundamentales de los computadores. Esto es sorprendente, pues para 1975, los fabricantes de semiconductores ya habían sacado al mercado los famosos procesadores de la serie 286.

Tomando como referencia la velocidad de los microprocesadores monolíticos de 1970, para el año 1978, la investigación y desarrollo de las empresas fabricantes de semiconductores monolíticos había logrado velocidades muy superiores. En la tabla 1 puede verse que la velocidad del microprocesador Pentium I que salió al mercado en 1994 era 12 veces mayor que la de su primer antecesor.

Las computadoras personales (PC's) aparecieron a finales de los años setenta, su aceptación y difusión entre las empresas y usuarios particulares fue lenta inicialmente. La primera versión muy popular de PC, fue la basada en el procesador 286. En ella se podían correr las primeras versiones de procesadores de palabra y hojas de cálculo para aplicaciones de oficina.
Durante los ochenta se implementaron versiones 386, 386SX, 386DX, 486S, 486DX, 486DX2. A mediados de los noventa se lanzaron al mercado internacional las primeras versiones de procesador Pentium y 586. A mediados de los noventa prácticamente cada empresa contaba ya con una PC.

Hoy en día existen diferentes versiones Pentium IV que trabajan a diferentes velocidades y estamos en la víspera de la aparición de versiones más poderosas.

Nicolás Negroponte (1992) pronosticaba que la tendencia para cada hogar en el futuro sería la existencia de al menos una PC y un sin numero de computadoras dedicadas para controlar todas las tareas caseras, como la temperatura y el inventario del refrigerador, la optimización de consumo de com bus ti ble, entre muchas otras aplicaciones.

"Las máquinas, escucharán, verán, hablarán y se comportarán como sirvientes. Cada individuo usará al día más de mil microprocesadores en diversas tareas, y es posible que en cada hogar existan unos mil procesadores".

Tabla 1. Comparacion entre procesadores AM D, CYRIX e IN TEL, (Esposito y Mastroiani, 1998)

\begin{tabular}{|c|c|c|c|c|}
\hline Familia de procesador & Fabricante & $\begin{array}{l}\text { Frecuencia } \\
\text { (MHZ) }\end{array}$ & $\begin{array}{l}\text { Velocidad del CPU } \\
\text { (Velocidad 2.0) }\end{array}$ & $\begin{array}{l}\text { Velocidad FPU } 1 \\
\text { (Velocidad 2.0) }\end{array}$ \\
\hline $3865 X$ & AMD & 40 & 54 & \\
\hline $386 \mathrm{DX}$ & AMD & 40 & 54 & \\
\hline $486 S X$ & INTEL & 25 & 84 & \\
\hline $4865 X$ & INTEL & 33 & 111 & \\
\hline $486 \mathrm{DX}$ & CYRIX & 33 & 112 & 320 \\
\hline $486 \mathrm{DX}$ & INTEL & 33 & 112 & 284 \\
\hline $486 \mathrm{DLC}$ & CYRIX & 40 & 130 & \\
\hline $486 \mathrm{~S}$ & CYRIX & 40 & 131 & \\
\hline $486 \mathrm{DX}$ & CYRIX & 40 & 132 & 355 \\
\hline $486 \mathrm{D} \times 2$ & AMD & 25 & 167 & 425 \\
\hline $486 \mathrm{DX} 2$ & CYRIX & 25 & 167 & 481 \\
\hline $486 \mathrm{D} \times 2$ & INTEL & 25 & 167 & 440 \\
\hline $486 \mathrm{D} \times 2$ & AMD & 33 & 223 & 588 \\
\hline $486 \mathrm{DX} 2$ & INTEL & 33 & 223 & 586 \\
\hline PENTIUM & INTEL & 60 & 346 & \\
\hline
\end{tabular}

${ }^{1}$ La velocidad de la Unidad de Punto Flotante (FPU, Floating Point Unit) se obtiene por cálculos en los que se considera los millones de instrucciones de punto flotante por segundo (MIPS) que pueden ser intercambiadas en las unidades de procesamiento y de memoria de una PC. Su utilidad puede verse al notar que la velocidad FPU de los procesadores 486DX2 de AMD, CYRIX e INTEL, es diferente, mientras que la velocidad de su CPU es igual en todos los casos. 
Su pronóstico empieza a cumplirse, los hogares "inteligentes" han sido una realidad desde el año 2003, ya que empresas como Samsung anunciaron la disponibilidad en el mercado de sus dispositivos inteligentes NetHome ${ }^{\circledR}$.

Actualmente se están desarrollando máquinas con sentido del tacto y existe el sueño entre los investigadores que las mismas lleguen a tener sentido común y entendimiento.

\section{Supercomputadoras aceleradas y computadoras cuánticas}

El 26 de junio del 2000 la Agencia de Noticias (Reuters), confirmó el lanzamiento al mercado de la computadora más rápida que existe hoy en el mundo, desarrollada por iniciativa del Departamento de Energía de los Estados Unidos de Norteamérica. (Department of Energy's Accelerated Stra te gic Com puting Ini tia tive, ASCI).

Esta computadora cuenta con una capacidad de 12.3 Teraflops y es utilizada para simular pruebas de armas nucleares. Una de sus características singulares es que usa clusters que agrupan 8192 microprocesadores de cobre.

Esta tecnología es extraordinaria, ya que los microprocesadores comerciales conocidos, usualmente se han fabricado de aleaciones de Silicio, Galio y otros semiconductores. Así pues, parece que las opiniones de los expertos se han quedado cortas, porque no sólo los semiconductores impulsaron el desarrollo de supercomputadores con velocidades y capacidades insospechadas, sino también las tecnologías de microprocesadores con base en conductores. Esto concuerda con el reporte hecho por Foner y Orlando (1987), desde fines de los ochenta, sobre las investigaciones en superconductores aplicables a computadoras hiper rápidas.

Continuando con esta carrera ascendente, Batelle (2000), en el reporte R\&D Funding Fore cast de enero del 2000 indicó que IBM en Yorktown Heights NY, asignó un financiamiento de 100 millones de dólares hasta el año 2004 para la obtención de una supercomputadora paralela basada en un conjunto de procesadores con capacidad de un PetaFLOP, es decir, 100x $10^{12}$ operaciones de punto flotante por segundo, lo que equivale a 100 teraflops.
Impulsando esta línea de investigación, la $\mathrm{ASCl}$ mantendrá proyectos en Súper cómputo Acelerado con un financiamiento de 320 millones de dólares hasta el año 2010.

Por otra parte, con el advenimiento de la electrónica óptica, cada vez parece más probable que en un fu turo mediato se convierta en realidad lo reportado por Zeilinger, a principios del 2000, en el sentido de que la tecnología sea conocida como "teleportación cuántica", la cual ya ha sido probada con fotones y tendrá su mayor aplicación en los sistemas de cómputo. De hecho, hoy en día ya existen las computadoras cuántica experimentales.

\section{Evolución de los sistemas operativos}

Paralelamente a la evolución del "hardware" de procesamiento y memoria que han permitido hoy en día tener sistemas con capacidad de millones de operaciones de punto flotante por segundo (Mflops y Teraflops), se han dedicado miles de horas hom bre en la elaboración y optimización de los sistemas operativos y del software de aplicación. Los costos de investigación, desarrollo, equipamiento y operación han sido extremadamente elevados. Aunque las inversiones para el desarrollo de sistemas computacionales son de alto riesgo, éste ha sido uno de los mejores negocios de los últimos tiempos. El punto fundamental que las promueve, es que las aplicaciones se pueden volver universales con una buena estrategia de mercado. La tabla 2 es un resumen de la historia de algunos de los sistemas operativos más importantes y de sus características (García et al., 2003), CSF ( a, b y c ).

\section{La digitalización de las comunicaciones y el fenómeno de la convergencia}

Desde los orígenes del hombre, una de sus necesidades primordiales y de su organización ha sido la comunicación. Para que esta exista, se requiere la conjunción de una serie de tecnologías, por lo que se trata de tecnologías integradas. La imprenta de tipo móvil, inventada por Gutemberg en el año 1450, fue el principal medio de comunicación por más de 450 años. Con el tiempo, se hizo necesario utilizar alguna tecnología por medio da la cual se pudieran enviar y recibir señales a través del espacio abierto. 
DOI: http://dx.doi.org/10.22201/fi.25940732e.2005.06n2.007

Hacia la convergencia total de las tecnologías de la información

Tabla 2. Resumen de la evolución de Sistemas 0 perativos y algunas de sus características

\begin{tabular}{|c|c|c|c|c|}
\hline Sistema Operativo & Año & Desarrollador & Gestión de procesos & Multiusuario \\
\hline Atlas & $1950-1960$ & Universidad de Manchester & Lotes & No \\
\hline Unix & 1969 & $\begin{array}{c}\text { Ritchie/Thompson Labs. } \\
\text { ATT\& \&ell }\end{array}$ & $\begin{array}{l}\text { Multiprogramado } \\
\text { T compartido }\end{array}$ & $\mathrm{Si}$ \\
\hline Unix PC Xenit & 1983 & $\begin{array}{c}\text { Ritchie/Thompson Labs. } \\
\text { ATT \& Bell }\end{array}$ & multiprogramado & No \\
\hline Sprite & 1984 & & multiprogramado & $\mathrm{Si}$ \\
\hline Merlin & 1984 & & Lotes & No \\
\hline Windows NT & 1985 & MIcrosoft & multiprogramado & $\mathrm{Si}$ \\
\hline Mach & 1986 & DARPA & multiprogramado & $\mathrm{Si}$ \\
\hline OS/2 ver. 1.0 & 1987 & Microsoft \& IBM & multiprogramado & No \\
\hline Os/2 ver. 1.1 & 1988 & Microsoft \& IBM & & $\mathrm{Si}$ \\
\hline Unix System V (redes) & 1989 & AT\&T & multiprogramado & $\mathrm{Si}$ \\
\hline Linux & 1991 & Linus Torvals & multiprogramado & No \\
\hline Windows 3.1 & 1992 & Microsoft & multiprogramado & No \\
\hline Unix 64 bits & 1993 & Novell - HP & multiprogramado & $\mathrm{Si}$ \\
\hline Windows 95/98 & $1995 / 98$ & Microsoft & multiprogramado & No \\
\hline Coyote & 1996 & Trinity College Dublin & Distribuido & $\mathrm{Si}$ \\
\hline Linux 2.2 & 1998 & $\begin{array}{c}\text { Linus Torvals y contribución } \\
\text { mundial de } \\
\text { programadores }\end{array}$ & multiprogramado & $\mathrm{Si}$ \\
\hline
\end{tabular}

El telégrafo inventado por Samuel Morse en 1844 fue el primer dispositivo que usó electricidad para enviar y recibir señales, pero estaba limitado a los códigos de combinaciones de puntos y guiones. Su prin ci pal limitación consistía en la necesidad de instalar pares de alambres entre el emisor y el recep tor (Volti, 1992).

La primera aplicación de las comunicaciones que aprovechó el principio de la propagación de las ondas electromagnéticas, cuya teoría fue desarrollada por James Clerk Maxwell en 1860, fue la transmisión del telégrafo inalámbrico de Guillermo Marconi en 1896.

La primera transmisión de voz por radio fue hecha por Fesseden en 1900, quien patentó su invento en 1905 y lo llamó transmisor heterodino. En 1918, Armstrong aplicó el bulbo triodo al receptor de radio y lo patentó con el nombre de superheterodino. La televisión experimental apareció en 1920. En 1936, la BBC de Londres hizo la primera transmisión de TV de "alta definición" usando un sistema llamado iconoscopio. Las primeras transmisiones de TV a color se hicieron en los Estados Unidos de Norteamérica en 1954. El primer radio transistorizado salió al mercado en 1955.

Hoy en día se entiende de forma coloquial que las "comunicaciones" son el proceso de manejo de señales para la transmisión de información a distancia. En este sentido, los primeros sistemas de comunicación como el radio, la televisión, el teléfono y el video se basaban en circuitos de procesamiento de electrónica analógica; esto es, trataban a la señal de una forma análoga a su forma física.

Posteriormente, la proliferación de los circuitos digitales permitió un manejo diferente para las señales de comunicaciones. La transmisión pudo realizarse por medio del muestreo y codificación de la información y datos en forma de bits. Los trenes de bits codificados según un protocolo preestablecido se conocen como señales digitales de alta resolución. 
En la gráfica mostrada en la figura 3 se puede ver que desde los años setenta el progreso de las tecnologías LSI aceleró la tendencia hacia la digitalización, lo que a la postre eliminó la barrera que había entre las computadoras y la tecnología de las comunicaciones.

Desde 1977, los directivos de la corporación industrial japonesa National Electric Corporation, (NEC), líder tecnológico en áreas como el radar, equipos de telecomunicaciones, materiales y dispositivos electrónicos, fueron los primeros en pronosticar la aparición de un fenómeno al que llamaron C\&C; es decir, la even tual fusión de las tecnologías de comunicaciones y la tecnologías de computo, debido a los progresos en las tecnologías de semiconductores (Saito, 1997).

Para disminuir el impacto del fenómeno de convergencia C\&C, los dirigentes de NEC diseñaron un Programa de Competencias Tecnológicas (Core Tech nol ogy Pro gram), en el cual se avistaban algunos puntos esenciales, tales como el de mantener la coherencia entre la administración tecnológica y la estrategia de negocios, identificar las familias de tecnologías nucleares (Core Technologies) y definir programas de inversión y de investigación para hacer frente a los retos del fenómeno.
Para no quedarse atrás, muchas otras empresas relacionadas con la electrónica en el ámbito mundial, prepararon diversas estrategias, relacionadas generalmente con mayores inversiones en investigación y desarrollo para enfrentar la situación.

Este proceso rindió sus frutos, ya que hacia el año de 1993, el fenómeno de convergencia presentó nuevas oportunidades de mercado para la industria electrónica norteamericana. Se consolidaron nuevas alianzas y dio inicio la era de los nuevos medios o mul ti me dia.

Un actor muy importante en este proceso de innovación continua, fue el gobierno norteamericano a través de sus financiamientos. Uno de los principales promotores ha sido sin duda el Instituto Nacional de Ciencia y Tecnología, (National Science \& Technology Institute, NIST) de los Estados Unidos de Norteamérica. Este organismo dispuso de fondos en 1993 por 68 millones de dólares para investigación, de 200 en 1994 y de 750 en 1997. Estas investigaciones financiadas por el gobierno, fueron un fuerte impulsor de la convergencia al promover la creación de la supercarretera de la información o Internet. El objetivo primario de esta red de alta velocidad era cubrir toda el área de los Estados Unidos de Norteamérica con propósitos de seguridad militar.

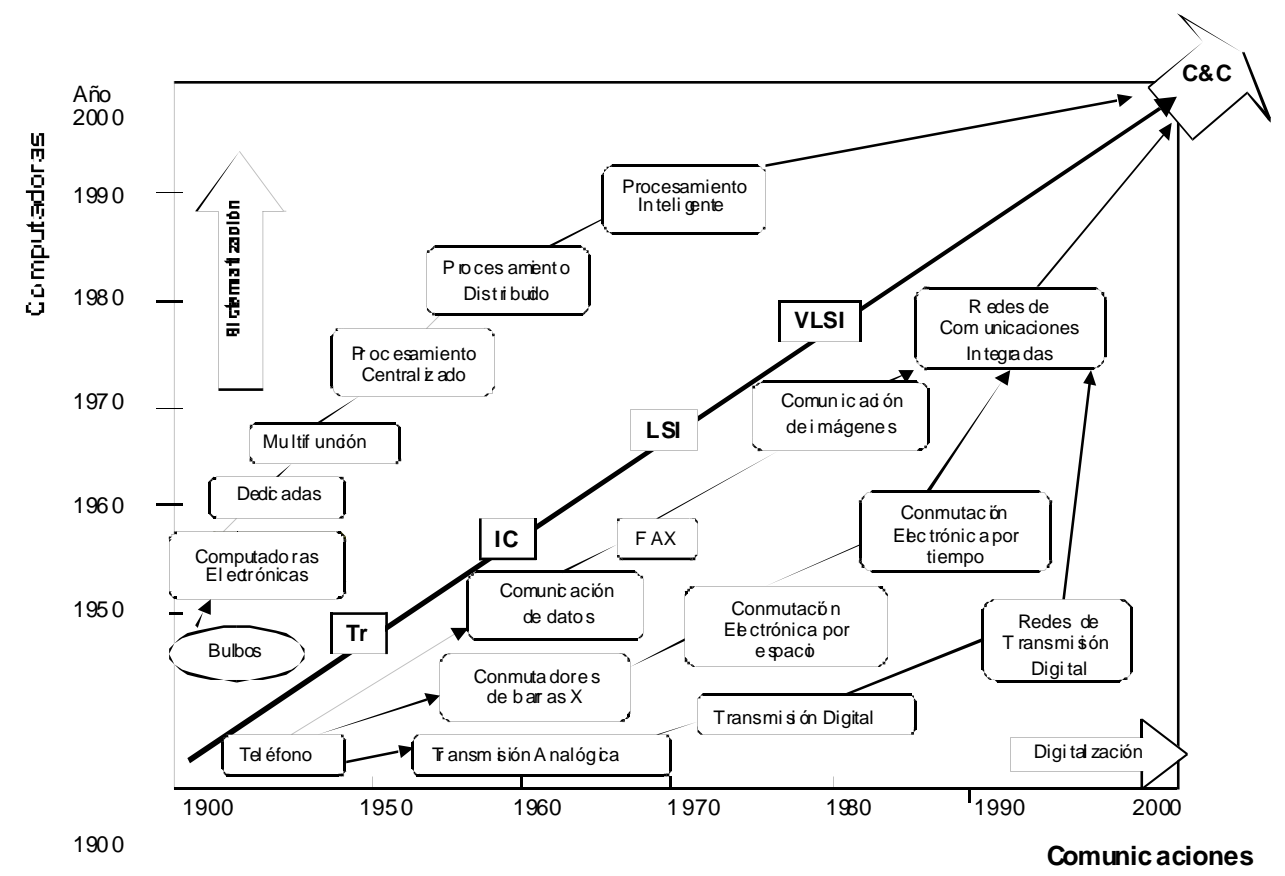

Figura 3. El fenómeno $C \& C$, convergencia de computadoras y comunicaciones (Saito, 1997) 
En poco tiempo se unieron al fenómeno de convergencia de las computadoras y las comunicaciones la electrónica de consumo y la in du stria del entretenimiento.

\section{Los protocolos de comunicaciones catalizadores de la convergencia}

En la figura 3 puede observarse que hasta los años ochenta con la tecnología de ultra escala de integración (ULSI), aparecieron en el mercado los procesadores inteligentes o microprocesadores. La disponibilidad de este hard ware era insuficiente para lograr sistemas integrales de comunicación, ya que la diversidad de fabricantes, sistemas y aplicaciones, dio origen a una diversidad enorme de protocolos propietarios.

De esta forma, los fabricantes de sistemas de manufactura, administrativos, de control, au tomatización y comunicaciones, disponían de sus propios protocolos en forma cerrada, lo que originó una gran lucha por el control de los mercados. Esto generó más problemas de los que resolvió, ya que los usuarios tenían que navegar en una diversidad muy compleja de sistemas que muchas veces no eran compatibles.

El concepto que resolvió el problema fue la normalización y estandarización de protocolos. Esto funcionó como catalizador, ya que incentivó la innovación y finalmente dio origen a redes de comunicación integradas.

El primer resultado de protocolos de alto impacto lo fue la red internacional Internet (In terna tional Net work), que es la denominación de una red de computadoras en el ámbito mundial que tienen en común el protocolo TCP/IP. Surgió en 1964 como una necesidad del gobierno de los Estados Unidos como estrategia militar du rante la guerra fría. Se trataba de encontrar los medios tecnológicos para establecer comunicación entre puntos geográficos distantes en caso de una guerra.

La empresa RAND Cor po ra tion encargada de la estrategia militar de EUA planteó la solución de crear una red de comunicaciones que no dependiera de un organismo cen tral, la cual estaría integrada por nodos o puntos de enlace de igual rango con la misma capacidad de originar, recibir y transmitir mensajes. En el caso de que alguno de los nodos dejara de funcionar, el resto de la red seguiría en operación. Los mensajes en esta red se dividirían en paquetes con una dirección propia.

En diciembre de 1969 ya existían 4 nodos en la red ARPANET, desarrollada por la agencia del Pentágono ARPA (Advanced Research Project Agency). En 1972 ya contaban con 37 nodos con líneas de transmisión de alta velocidad. La ventaja de la red ARPANET era que no importaba el tamaño ni el tipo de maquinas que estuvieran interconectadas mientras cumplieran con el protocolo NCP (Network Control Proto col), que fue establecido originalmente. En esa época, diferentes fabricantes establecieron sus propios proto colos para sus redes de comunicación. Así nacieron los estándares Ether net, FDDI y X.25 entre muchos otros.

En 1974, se cambió el NCP por un nuevo protocolo estándar más sofisticado llamado TCP/IP (Transmission Control Protocol/Internet Pro to col), cuyos inventores fueron Vint Cerf y Bob Kahn. La ventaja de este protocolo era que convertiría los mensajes en cadenas de paquetes en el nodo de origen y los volvería a ensamblar en el nodo destino. EI TCP/IP además podría rutear paquetes de mensajes en redes que trabajaban con protocolos diferentes.

En 1989, México ingresó a Internet a través de NSFNET y la red BITNET que permitían acceso a recursos de información existentes en México y en el mundo.

En 1992 se fundó ISOC (Internet So ci ety)para coordinar el uso de las tecnologías existentes en beneficio de todos los usuarios. Es así como se desarrolló el World Wide Web (WWW) que permitió un acercamiento más fácil a través del hipertexto a todos los recursos de Internet, también dando una muestra de la transmisión de audio y video en tiempo real a través de la red.

El crecimiento de la red se volvió exponencial con el uso de los exploradores que son programas especialmente diseñados para navegar en forma óptima. El primero de ellos que aprovechó la gran capacidad del WWW, fue denominado Mosaic, desarrollado por la Universidad de Illinois Urban-Champagne.

En 1995, los sistemas de servicios vía modem ofrecidos por compañías como Compuserve, Prodigy y Genie comenzaron a ofrecer servicios por Internet. A fi na les de los noventa, el navegador de Netscape obtuvo el mayor índice de ganancias 
jamás obtenido en Wall Street. Hoy en día el InternetExplorer de Microsoft do mina el mercado amenazando con un dominio monopólico mundial de esa firma para los sistemas de oficina.

Tal vez una de las aplicaciones hasta hoy más sobresalientes del Internet es el correo electrónico (email), el cual permite a la gente y a las organizaciones enviar y recibir mensajes usando su computadora personal, un módem y una línea telefónica. El correo electrónico se encuentra basado en el concepto de dirección. La dirección email provee toda la información específica para comunicarse con cualquier parte del mundo.

\section{La era de la información multimedia}

Un resultado espectacular del proceso continuo de digitalización, el cual trabaja en conjunto con la disponibilidad de Internet es la explosión multimedia del día de hoy. La tecnología digital traduce información de virtualmente cualquier medio a código binario permitiendo que sea transmitida por medio de protocolos y códigos comunes y procesada por computadoras.

Los servicios por Internet, junto con la electrónica de consumo, se han convertido en dos áreas de inmenso potencial para las empresas de la industria mundial electrónica. Las empresas norteamericanas se mantienen al frente, desarrollando y suministrando nuevas herramientas para impulsar la productividad en el trabajo, simplificar los procesos de negocios, modificar los patrones de educación y establecer nuevos sistemas de salud. Los sistemas multimedia han atraído una diversidad de empresas, una muestra de ellas puede verse en la tabla 2.

La convergencia $C \& C$ se ha extendido a otros cam pos, tales como el del entretenimiento, debido al enlace comercial de las empresas proveedoras de servicios telefónicos y las compañías de televisión por cable. Algunos de los factores técnicos que han contribuido en este proceso han sido los siguientes:

- Las redes digitales se han convertido en manejadoras de software en forma incre mental, esto ha impulsado a las empresas a adquirir y desarrollar más capacidad de software bajo contrato.
- El costo de las memorias ha disminuido constantemente. Los requerimientos de memoria para el manejo masivo de videos y películas son elevados, por lo que sólo disponiendo de memorias más baratas se posibilitará el producto "películas sobre demanda"

- El costo de las transmisiones disminuye constantemente. El equipo de multiplexaje se ha mejorado cada año desde que apareció la fibra óptica, hace aproximadamente unos veinte años, logrando cada vez mayores anchos de banda. La capacidad de transmisión se ve hoy en día como un commodity ó producto de alto consumo.

- Las capacidades de las redes inalámbricas se han expandido tremendamente

Las compañías de televisión por cable están instalando gradualmente cable de fibra óptica en lugar de cable coaxial. También se están reemplazando las redes de pares trenzados de cobre usadas tradicionalmente por las compañías telefónicas. Hoy en día una empresa de televisión por cable puede convertirse en una empresa de servicio telefónico y viceversa.

La expansión de los sistemas satelitales de comunicación en conjunto con el dominio de las nuevas tecnologías de soft ware y la integración de los recursos tecnológicos de transmisión están provocando una gran competencia en las empresas de telecomunicaciones y de Soft ware.

A través de protocolos de comunicación como el ATM o el IP /WDM, hoy en día están disponibles servicios convergentes que incluyen el manejo de voz, datos, video y servicios de redes con buena calidad de servicio (Vega et al., 2003).

Un ejemplo espectacular de estas tecnologías multimedia convergentes salió al mercado a principios del año 2000 y es el teléfono celular con capacidad de acceso a Internet. Este es un Sistema de Comunicación Per sonal (PCS) que hace uso de la supercarretera de la información, aprovechando las capacidades de alta velocidad, potencia, ancho de banda y confiabilidad de las redes de comunicación satelital.

En el contexto descrito en los apartados anteriores, las oportunidades para servicios 
orientados a negocios se han expandido de manera sustancial. Actualmente, disponemos de los sistemas de video conferencia de escritorio, de dispositivos digitales de uso personal, soft ware de trabajo en grupo, pizarrones electrónicos y otros servicios y productos multimedia que están conformando lo que se ha dado en llamar la nueva ola de herramientas de productividad en los negocios.

Microsoft está trabajando en software para interfase de teléfonos con diversos dispositivos de oficina con la capacidad de acceso a servicios avanzados de redes. AT\&T ha introducido nuevos sistemas de mensajería integrada, por lo que en el futuro los mensajes de oficina tendrán más contenido multimedia: se enviará y recibirá voz, texto y video. De hecho, esto ya se hace frecuentemente utilizando las capacidades de Internet a través del soft ware para redes internas corporativas Intranet. Otra área de crecimiento espectacular ha sido la aplicación de los microprocesadores en los
Asistentes Digitales Personales (PDA), tales como agendas, comunicadores personales y la computadora tipo pluma.

Por otra parte, el fenómeno de trabajadores móviles está creando una demanda cada vez más creciente para la electrónica portátil. En suma, los sistemas mul ti me dia están permeando el mercado con un número tremendo de aplicaciones. En la tabla 3 puede verse una pequeña fracción de las empresas relacionadas hoy en día con la producción de sistemas mul ti me dia.

En poco tiempo se espera el desarrollo del mercado del "videoservidor" con capacidad de almacenar cientos de películas comprimidas digitalmente y transmitir su contenido a casas, escuelas y negocios. En este mercado se encuentran compitiendo Hewllet Packard, Silicon Graphics, nCube, Digital Equipment, IBM y ATT. También empresas de soft ware como Or a cle y Microsoft se beneficiarán de esta tecnología del video-servidor y sus productos derivados.

Tabla 3. Empresas relacionadas con sistemas multimedia

\begin{tabular}{cccc}
\hline $\begin{array}{c}\text { Empresas de electrónica } \\
\text { establecidas }\end{array}$ & $\begin{array}{c}\text { Nuevas empresas de } \\
\text { multimedia }\end{array}$ & $\begin{array}{c}\text { Empresas proveedoras de } \\
\text { servicios de Redes }\end{array}$ & $\begin{array}{c}\text { Otras empresas con } \\
\text { potencial multimedia }\end{array}$ \\
\hline Apple & 3DO & AT\&T & Acclain Entertaiment \\
Broderbund & Books that Work & Bell Atlantic & Atari \\
Cisco Systems & Bell Canada & Commodore \\
Compton's New & Bedia & Disney \\
Crystal Dynamics & General Magic & MCC/First & Cities Electronic Arts \\
Hewlett Packard & Consortium & Kodak \\
IBM & Ikonic Interactive & Nynex & LucasArts: Industrial \\
Kaleida Labs & Pacific Bell & Light \& Magic \\
Intel & Media Vision & Sierra on Line & Next \\
Lotus & PF Magic & Sothwest Bell & Nintendo \\
Matsushita & Pop Rocket & TBS & Paramount \\
Microsoft & Rocket Science & TCl & Sega \\
& Games & & Time Warner \\
NEC & Sanctuary Woods & United Video & \\
\hline
\end{tabular}

continúa... 
Tabla 3. E mpresas relacionadas con sistemas multi media (continuación...)

\begin{tabular}{|c|c|c|c|}
\hline $\begin{array}{c}\text { Empresas de electrónica } \\
\text { establecidas }\end{array}$ & $\begin{array}{c}\text { Nuevas empresas de } \\
\text { multimedia }\end{array}$ & $\begin{array}{c}\text { Empresas proveedoras de } \\
\text { servicios de Redes }\end{array}$ & $\begin{array}{l}\text { Otras empresas con } \\
\text { potencial multimedia }\end{array}$ \\
\hline Philips & Spectrum Holobyte & Satellite Group & \\
\hline \multirow[t]{2}{*}{ Scientific Atlanta } & Virgin Interactive & US West & \\
\hline & Entertaiment & & \\
\hline Silicon Graphics & & Viacom & \\
\hline \multicolumn{4}{|l|}{ Sony } \\
\hline \multicolumn{4}{|l|}{ Sun Microsystems } \\
\hline Toshiba & & & \\
\hline
\end{tabular}

\section{Resumen}

Las computadoras se aplicaron en los años setenta del siglo pasado, principalmente como equipos terminales en las redes de sistemas de comunicación. Algunos computadores específicos se utilizaron en los sistemas satelitales de transporte y en los sistemas electromecánicos de conmutación. La información manejada por los sistemas digitales era fundamentalmente voz (telefonía) y datos. El servicio era de mala calidad, ya que frecuentemente se presentaban interrupciones, pérdidas de información y se requería de procedimientos de chequeo intensivo y retransmisión, por lo cual, la comunicación era de baja eficiencia. Los sistemas operativos de la época estaban enfocados a la gestión de procesos por lotes para equipos terminales individuales.

En los años ochenta la diferencia sustancial fue que los computadores se utilizaron como redes terminales cuyo acceso a los sistemas de datos de conmutación y transporte se realizaba mediante equipos computarizados PBX. La información manejada por los sistemas digitales era fundamentalmente datos y voz. La calidad fue un poco mejor, debido a la mayor velocidad y capacidad de los sistemas de conmutación al utilizar los procesadores más poderosos de las tecnologías VLSI. Los protocolos de comunicación usados entre los distintos computadores que formaban alguna red de aplicación externa de tipo médico, control, instrumentación, manufactura, etcétera, eran del tipo cerrado. Si se corría la aplicación y se quería comunicar a alguna localidad distante geográficamente, había que seleccionar los datos requeridos, transportarla a alguna computadora terminal de los sistemas de comunicación de acarreo (car ri ers) y enviarla por medio de protocolos que hacían la función de transporte. La comunicación era de una calidad de servicio y alcances limitados.

En la primera parte de los años noventa fueron utilizados los medios de transmisión de banda ancha, específicamente la fibra óptica, que permitió incluir imágenes en los sistemas de comunicación, aunque en un principio de muy baja calidad. Tanto las computadoras de los equipos terminales, de los sistemas PBX como de los sistemas de conmutación, utilizaban procesadores ULSI. Aparecieron los sistemas operativos Mach, Windows NT y Uníx System V para redes, permitiendo una mejor comunicación en las redes terminales. A finales de los noventas, la ola de innovación tecnológica en los distintos constituyentes de los sistemas de comunicación permitieron comunicaciones de alta calidad de servicio, incluyendo voz, imágenes y video, telefonía celular, equipos terminales multimedia y sistemas de acceso, transporte y conmutación de banda muy ancha y alta velocidad. Los protocolos de comunicación para las redes de comunicación de enlace entre puntos apartados geográficamente estuvieron a punto y se generaron estándares para conexiones abiertas a partir de los computadores de aplicación de los usuarios. 


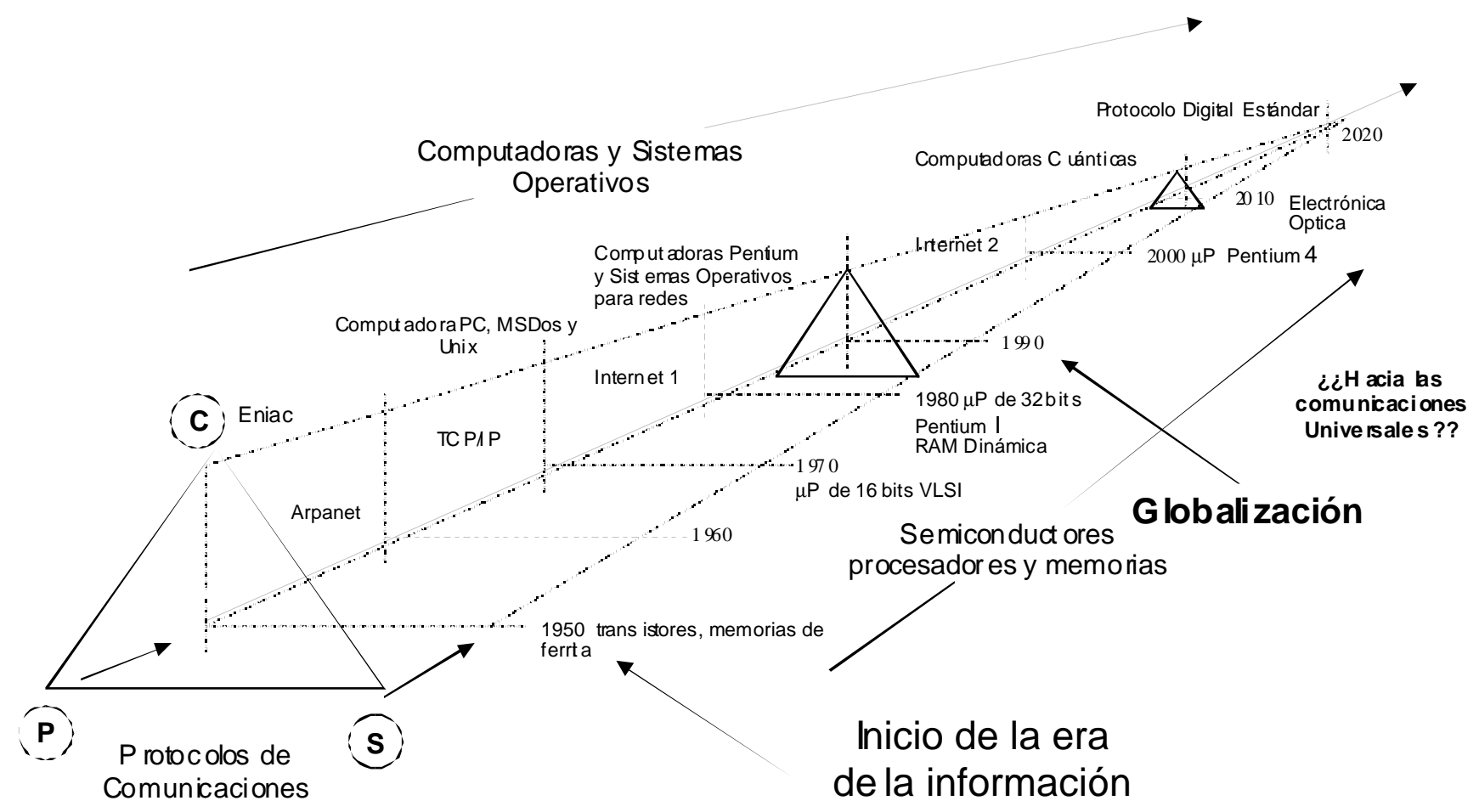

Figura 4. La convergencia total de las tecnologías de la información (Elaboración propia)

\section{Conclusiones}

A partir de la discusión de los diferentes apartados en los que hemos revisado brevemente cuál ha sido la evolución tecnológica de las TI y tomando como ejes impulsores de la innovación tecnológica: 1) los dispositivos semiconductores, procesadores y memorias (S); 2 ) las computadoras y sus sistemas operativos $(C) ;$ y 3 ) los protocolos de comunicaciones $(P)$, es posible construir la figura 4, la cual muestra la clara posibilidad, de que en un par de décadas la tendencia de la dinámica de las vari ables nos lleve a la convergencia total de la tecnologías de la información.

Analizando la figura, es evidente que con la integración, en el sentido descrito por lansati (op.cit.), de las tecnologías de la información disponibles para la década de los 90's del siglo pasado, se sentaron las bases para la globalización. A partir de entonces, la humanidad ha dispuesto de tecnología de comunicación de voz, datos e imágenes con alta calidad de servicio entre puntos distantes geográficamente de la tierra.

La convergencia $C \& C$ pronosticada en los 70 's no sólo fue cumplida a plenitud, sino que fue rebasada en cuanto a sus alcances. Para el futuro se considera que los sistemas de protocolos estandarizados prevalecerán generando sistemas abiertos de comunicación absoluta. Esto podría abrir el paso a los sistemas de tele transportación cuántica. Hoy en día los sistemas computacionales tienen enormes capacidades de procesamiento y memoria y se están desarrollando por medio de la electrónica óptica. Los nuevos protocolos de comunicación IP/WDM están buscando la transmisión de información sobre medios ópticos con la mira de usar el ancho de banda infinito de la luz.

El ejercicio prospectivo de ex tender la tendencia de las variables, nos señala que hacia el año 2020 podemos esperar la convergencia absoluta de las tecnologías de la información (S, C\&P). Esto promoverá la definición de nuevos estándares mundiales y dará origen a las tecnologías estandarizadas de la información o tecnologías de información estándar (TIE) (Vega op. cit.). Prob a blemente estemos en camino hacia un sistema de comunicaciones universales en el que por medio de dispositivos electro-ópticos sea posible establecer contacto con los tripulantes de las colonias humanas que habitarán las futuras estaciones espaciales. 
Todo avance tecnológico tiene impactos. Las tecnologías de la información integradas en sistemas de comunicaciones abiertos absolutos seguramente impactarán a la sociedad y al género humano. Consecuentemente se impulsarán to dos los cam pos de las ciencias, como en el caso de la bioingeniería, en que las supercomputadoras ayudarán a la finalización de extensiones del proyecto "Genoma Humano" y a la explotación de la información que de ahí se derive. Se desarrollarán nuevos dispositivos militares de defensa y ataque. También se promoverá el desarrollo de nuevos vehículos y estaciones espaciales.

En el ámbito casero, la mayor parte de los dispositivos, ostentarán inteligencia y tal como lo predijo Negroponte una década atrás, todo apunta a que la computadora será un dispositivo omnipresente.

El gradiente del cambio tecnológico se muestra con una aceleración vertiginosa. Los ciclos de vida de los productos electrónicos se están reduciendo de años a meses y probablemente en algunos años serán de apenas unos días. La convergencia absoluta $S, C \& P$, generará nuevos dispositivos cibernéticos, los cuales tendrán influencia y con trol en todos los cam pos de la vida humana.

Una tarea pendiente es que los individuos, sus organizaciones y sus sociedades, se deben preparar para asimilar los cambios por venir. Rivers (2002), sostiene que el progreso tecnológico se sustenta en su propia mitología, la cual extiende y perpetúa la idea de progreso indefinidamente en el fu turo.

Dependerá de nosotros que el progreso tecnológico se convierta en progreso humano, éste enorme problema será tarea de todos y seguramente no será fácil resolverlo.

\section{Referencias}

Batelle (2000). R\&D Funding Fore cast, January.

(a).Computer Story CSF, Sistemas Operativos, Línea de tiempo Uníx:

http://www.comsto.org/so/linux.htm

(b).Computer Story CSF, Sistemas Operativos, Uníx, el fenómeno:

http://www.comsto.org/so/unix.htm

(c) .Computer Story CSF, Sistemas Operativos, OS2: el divorcio entre IBM y Microsoft:

http://www.comsto.org/so/os2.htm
Esposito E. y Mastroiani M. (1998), Tech nological Evolution of Personal Computers and Market Implications. Technological Forecasting and So cial Change 59, pp. 235-254.

Fiap (1998). La alternativa social: la sociedad global del conocimiento. Revista Digital Desen Reda, No. 2, Año 2.

. http//:www.arrakis.es/fiap/revi2art6.htm

Foner S. y Orlando T.P. (1987). Superconductors, the Long Road Ahead. Technology Review, Febru ary-March, pp. 36-47.

Garcia L., Peralta L. y Fernández S. (2003). Sistemas Operativos. Un paseo por la histo ria, http://spisa.act.uji.es/ peralta/os/

Iansiti M. y West J. (1997). Tech nolo gy Inte gra tion: Turning Great Research into Great Products. Harvard Busi ness Review, May-June, pp. 69-79.

Modelski G. (2001). "What causes K - waves? Technological Forecasting \& Social Change, Vol. 68, pp. 75-80. Elsevier Science Inc.

Negroponte N. (1992). Machine Dreams. Technology Review, January, pp. 33-39.

Reuters,http://dailynews.netsc...ntz0725-stor $y$-bcnewstechibmdc.html

Rivers T.J. (2002). Progress and Technology: their Interdependency. Tech nology in Society, Vol. 24, pp. 503-522.

Saito F. (1997). Managing Technology development at NEC Corporation. International Journal of Technology M anagement, Vol 14, No 2/3/4, pp. 196-207.

Smith H. y Antoniadis D. (1990). Seeking a Radically New Electronics. Technology Review, April, pp. 26-49.

Volti R. (1992). Society and Tech nolog ical Change. $2^{\text {nd }}$. Ed. St. Martin Press, NY.

Vega G.L.R., Avilés F. y Montalvo T.M. (2003). Evolución y evaluación tecnológica de los protocolos de comunicaciones. Revista Ingeniería, Investigación y Tecnología, Vol.4, No. 2, abril-junio, pp. 71-81.

Zeilinger A. (2000). Quantum Teleportation. Scien tificA merican, Vol. 282, No. 4, April, pp. $32-42$. 


\section{Bibliografía sugerida}

Ayres R.U. (1990). TechnologicalTransformations and Long Waves. Part I. Technological Forecasting and Social Change. Vol. 37, pp. 1-37. Elsevier Publishing Co.

Ayres R.U. (1990). TechnologicalTransformations and Long Waves. Part II. Technological
Forecasting and Social Change, Vol. 36, pp. 111-137, Elsevier Publishing Co.

Gross Neil editor (1996). Gilding the Ultimate Chip in Developments to Watch. Business Week, Octo ber 14, p. 88.

Lemonick M.D. (1995). Future Tech is Now. Times, July 17, pp. 36-39.

\section{Semblanza del autor}

Luis Roberto Vega-González. Es ingeniero mecánico electricista por la Facultad de Ingeniería de la UNAM. Obtuvo la maestría en ingeniería de sistemas en el área de planeación por la DEPFI-UNAM y la maestría en administración de las organizaciones por la División de Estudios de Posgrado de la Facultad de Contaduría y Administración de la UNAM. Asimismo, se especializó en gestión de la innovación tecnológica por el Centro para la Innovación Tecnológica y la FCyA de la UNAM. Durante más de veinte años colaboró en diversas firmas nacionales e internacionales con proyectos en las áreas de instrumentación, control y potencia. Actualmente es coordinador de vinculación y gestión tecnológica del Centro de Ciencias Aplicadas y Desarrollo Tecnológico (CCADET) de la UNAM. 